\title{
New group-V elemental bilayers: A tunable structure model with four-, six-, and eight-atom rings
}

\author{
Xiangru Kong, ${ }^{1, *}$ Linyang Li, ${ }^{2, \dagger}$ Ortwin Leenaerts, ${ }^{2}$ Xiong-Jun Liu, ${ }^{1}$ and François M. Peeters ${ }^{2}$ \\ ${ }^{1}$ International Center for Quantum Materials, School of Physics, Peking University, Beijing 100871, China \\ and Collaborative Innovation Center of Quantum Matter, Beijing 100871, China \\ ${ }^{2}$ Department of Physics, University of Antwerp, Groenenborgerlaan 171, B-2020 Antwerp, Belgium
}

(Received 24 March 2017; published 13 July 2017)

\begin{abstract}
Two-dimensional group- $\mathrm{V}$ elemental materials have attracted widespread attention due to their nonzero band gap while displaying high electron mobility. Using first-principles calculations, we propose a series of new elemental bilayers with group-V elements ( $\mathrm{Bi}, \mathrm{Sb}, \mathrm{As}$ ). Our study reveals the dynamical stability of four-, six-, and eight-atom ring structures, demonstrating their possible coexistence in such bilayer systems. The proposed structures for $\mathrm{Sb}$ and $\mathrm{As}$ are large-gap semiconductors that are potentially interesting for applications in future nanodevices. The Bi structures have nontrivial topological properties with a direct nontrivial band gap. The nontrivial gap is shown to arise from a band inversion at the Brillouin zone center due to the strong intrinsic spin-orbit coupling in $\mathrm{Bi}$ atoms. Moreover, we demonstrate the possibility of tuning the properties of these materials by enhancing the ratio of six-atom rings to four- and eight-atom rings, which results in wider nontrivial band gaps and lower formation energies.
\end{abstract}

DOI: 10.1103/PhysRevB.96.035123

\section{INTRODUCTION}

The story of two-dimensional (2D) materials begins with the successful exfoliation of graphene from graphite [1]. Two-dimensional materials are usually defined as crystalline materials consisting of a single or a few layers of atoms. The unusual physical properties, caused by dimensional restrictions, lead researchers to study these materials for possible use in applications and future nanodevices [2]. The search for other 2D materials besides graphene is an ongoing field of research. In analogy to graphene, other group IV elements also form 2D hexagonal structures, such as silicene [3-6], germanene [7,8], and stanene [9], and have been successfully synthesized on different substrates. Similar structures could also be observed for the $2 \mathrm{D}$ group- $\mathrm{V}$ elemental structures. In theory, buckled hexagonal honeycomb bilayers of group-V elements are also stable and favorable in energy [10]. For example, hexagonal $\mathrm{Bi}(111)$ bilayers [11-14] have been experimentally synthesized on $\mathrm{Bi}_{2} \mathrm{Te}_{3}$ or $\mathrm{Bi}_{2} \mathrm{Se}_{3}$ surfaces [15-17]. In this connection, the successful growth of singlelayer blue phosphorus has attracted widespread attention to the group-V elemental bilayers due to their nonzero band gap and high electron mobility [18]. There have also been many suggestions for other 2D stable carbon allotropes [19,20], such as phagraphene [21] and graphyne [22,23], and some of them have been successfully created or can be found as defects in graphene. The physical properties of such crystalline materials mainly originate from the underlying symmetry of the crystal structure. Therefore, it is interesting to study $2 \mathrm{D}$ crystal structures with different symmetries. Recently, some theoretical works have studied 2D group-V structures with four-atom and eight-atom rings on a square lattice [24,25]. However, the formation energy of these bilayer structures is relatively high, which makes it difficult to realize them

\footnotetext{
*kongxru@pku.edu.cn

†linyang.li@uantwerpen.be
}

in experiments. It is thus an interesting question how such materials can be made more stable.

One of the most intriguing properties of some 2D materials is their nontrivial band topology. Two-dimensional topological insulators with time-reversal symmetry, also known as quantum spin Hall (QSH) insulators, are a very important set of 2D materials [26-29]. Graphene was the first proposal for such a topological insulator, but its negligible nontrivial band gap makes it impossible to observe the QSH effect [29,30]. In experiments, the QSH effect has been obeserved in $\mathrm{HgTe} / \mathrm{CdTe}$ and $\mathrm{InAs} / \mathrm{GaSb}$ quantum wells [31-33], but the small bulk gap arising from weak spin-orbit coupling (SOC) makes the operating temperature very low and this limits its further applications [34-38]. To realize 2D topological insulators with a large band gap, most studies have focused on some heavy elements, such as $\mathrm{Bi}$, which exhibit a strong SOC effect. The largest nontrivial bulk gap $(1.08 \mathrm{eV})$ is found in the $\mathrm{Bi}_{2} \mathrm{~F}_{2}$ bilayer. The huge SOC gap in this material originates from the $\mathrm{Bi} p_{x}$ and $p_{y}$ orbitals $[39,40]$. But also hexagonal $\mathrm{Bi}(111)$ bilayers have been realized and their time-reversal symmetry-protected edge states have been observed [15]. However, their topological nature is still debated. The search for other Bi-based QSH insulators is therefore interesting.

In this work, we propose a new structure model with fouratom, six-atom, and eight-atom rings for the group-V elements: $\mathrm{Bi}, \mathrm{Sb}$, and As. The formation energy of these proposed structures is lower than those of other reported 2D group- $\mathrm{V}$ structures containing four- and eight-atom rings [24,25]. We find that their phonon spectra contain no imaginary frequency modes, indicating their dynamical stability. In the case of $\mathrm{Bi}$, the calculated band structure suggests nontrivial topological properties with a direct nontrivial bulk gap, resulting from a band inversion at the $\Gamma$ point. The proposed $\mathrm{Sb}$ and As bilayers show large indirect band gaps with SOC, but these band gaps are trivial. We demonstrate that the properties of the proposed structures can be tuned by the number of six-atom rings. For $\mathrm{Bi}$, we show that the formation energy can be decreased while retaining the topologically nontrivial properties. 
The paper is organized as follows: in Sec. II we briefly introduce the computational methods; in Sec. III we discuss the results on structure and stability (Sec. III A), electronic band structures (Sec. III B), topological properties (Sec. III C), and structural tunability (Sec. III D); and in Sec. IV we summarize and conclude the paper.

\section{COMPUTATIONAL METHODS}

Our first-principles calculations are based on density functional theory with the projector augmented wave method as implemented in the Vienna ab initio simulation package [41-43]. The generalized gradient approximation in the form proposed by Perdew, Burke, and Ernzerhof (PBE) [44] was chosen as the electron exchange-correlation functional. The structure relaxation including the atomic positions and lattice vectors was performed by the conjugate gradient scheme until the maximum force on each atom was less than $0.01 \mathrm{eV} / \AA$. The energy cutoff of the plane waves was set to $500 \mathrm{eV}$, with an energy precision of $10^{-5} \mathrm{eV}$. The Brillouin zone was sampled by using a $13 \times 7 \times 1 \Gamma$-centered Monkhorst-Pack grid. At the same time, to give a more reliable calculation of electronic band structures, the screened Heyd-Scuseria-Ernzerhof hybrid functional method (HSE06) [45,46] was also used with a $7 \times 3 \times 1 \Gamma$-centered Monkhorst-Pack grid for Brillouinzone integration. The main electronic band structures discussed in the article are obtained at the HSE06 level. Phonon frequencies are calculated by the finite displacement method with the PHONOPY code [47].

The $Z_{2}$ topological invariants were obtained by calculating the Wannier charge centers (WCCs) and tracking the largest gap in the spectrum of the WCCs [48], which is equivalent to computation of the Wilson loop [49]. The explicit numerical computations were done with the Z2PACK code [50], which combines the $a b$ initio calculations with the WANNIER90 code [51]. The surface state calculations are illustrated with an effective tight-binding Hamiltonian generated from the first-principles Wannier functions. The $p_{x}, p_{y}$, and $p_{z}$ orbitals of the $\mathrm{Bi}$ atoms from the first-principles wave functions are used as the initial trial orbitals. The iterative Greens function method [52] was used with the software package Wannier_tools [53].

\section{RESULTS}

\section{A. Structure and stability}

Due to the similarity of the proposed structures for the various investigated elements, we mainly focus on the structure model of $\mathrm{Bi}$ in this section. An example of this structure is shown in Fig. 1. Its lattice is rectangular, which is different from the hexagonal lattice of $\mathrm{Bi}(111)$ bilayers [11-14] and the square lattice of the recently proposed Bi bilayers consisting of four- and eight-atom rings [24,25]. The space group of the proposed orthorhombic crystals is $P c c m$ (or $D_{2 h}^{3}$ ). There are a twofold rotation, mirror, and inversion symmetry in this structure. Along the $x$ direction, there are two kinds of arrangements of atomic rings. One is formed by the line along the center of four (eight)-atom rings, while the other is along the center of the six-atom rings, as indicated by the dashed blue line in Fig. 1(a). The two arrangements of
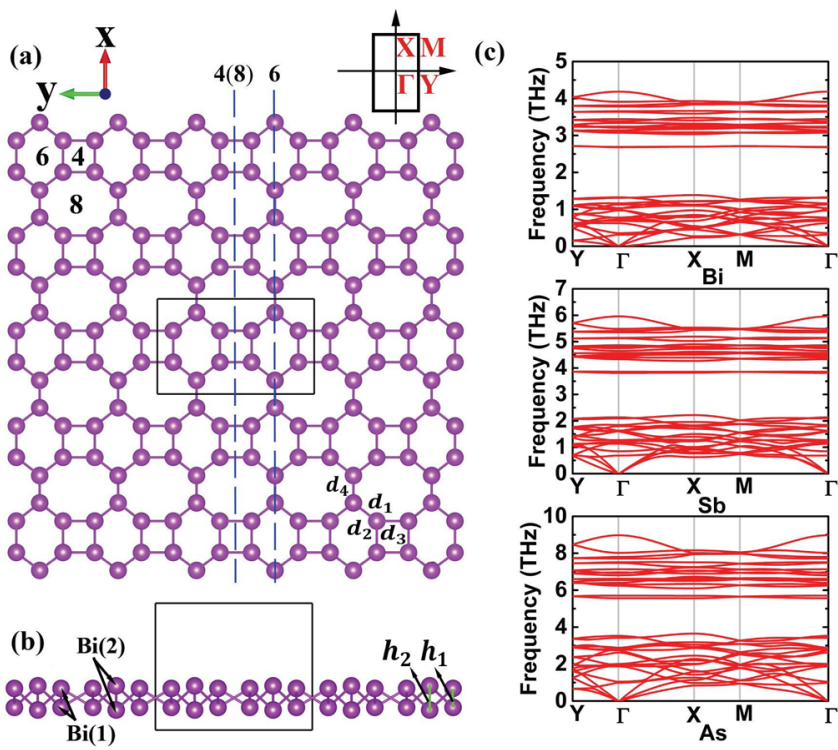

FIG. 1. (a) Top view of the 4(8)-6 Bi bilayer: the rectangle indicates the unit cell, and dashed blue lines show the four (eight)center-connected and six-center-connected lines. Upper-right inset: Brillouin zone with the time-reversal invariant momenta. (b) Side view of the 4(8)-6 Bi bilayer. (c) Phonon spectra of the 4(8)-6 Bi, Sb, and As bilayers.

atomic rings alternate along the $y$ direction and form a new type of Bi bilayer. Regarding the number of atomic rings, one four-atom ring corresponds to one eight-atom ring and one six-atom ring. Since the four-atom rings always come in pairs with the eight-atom rings, our structure is denoted a 4(8)-6 Bi bilayer in the following. As demonstrated below, such structures can be easily tuned by including more hexagons. A similar structure model can be applied to $\mathrm{Sb}$ and As.

The optimized structure parameters for the 4(8)-6 bilayers of $\mathrm{Bi}, \mathrm{Sb}$, and As are listed in Table I. Due to the similarity of these 4(8)-6 bilayers, we focus on the Bi bilayer first. The lattice constant $a(b)$ in the $x(y)$ direction of $\mathrm{Bi}$ is $7.918 \AA$ $(13.050 \AA)$. Although the lattice of the 4(8)-6 bilayer has a different symmetry than the hexagonal and square Bi bilayers, the local arrangement of the neighboring atoms is similar [11-14,24,25]. One Bi atom forms a bond with three other $\mathrm{Bi}$ atoms, which are all above or below the position of the $\mathrm{Bi}$ atom in the $z$ direction. However, while there is only one kind of Bi atom (one Wyckoff position) in hexagonal and square

TABLE I. Optimized structure parameters of 4(8)-6 Bi, Sb, and As bilayers. $a(b)$ is the lattice constant in the $x(y)$ direction, $h_{1}$ and $h_{2}$ are the buckling heights as shown in Fig. 1(b), and $d_{1,2,3,4}$ denote the different bond lengths shown in Fig. 1(a). $\Delta E$ is the formation energy defined by Eq. (1).

\begin{tabular}{|c|c|c|c|c|c|c|c|c|}
\hline $\begin{array}{l}a \\
(\AA)\end{array}$ & $\begin{array}{l}b \\
(\AA)\end{array}$ & $\begin{array}{l}h_{1} \\
(\AA)\end{array}$ & $\begin{array}{l}h_{2} \\
(\AA)\end{array}$ & $\begin{array}{l}d_{1} \\
(\AA)\end{array}$ & $\begin{array}{l}d_{2} \\
(\AA)\end{array}$ & $\begin{array}{l}d_{3} \\
(\AA)\end{array}$ & $\begin{array}{l}d_{4} \\
(\AA)\end{array}$ & $\begin{array}{c}\Delta E \\
\text { (meV/atom) }\end{array}$ \\
\hline & & & & & & & & 52 \\
\hline & & & 1. & & & & 2. & 58.1 \\
\hline 6.5 & .90 & $?$ & 150 & 25 & & & & 71.9 \\
\hline
\end{tabular}


Bi bilayers, there are two kinds of Bi atoms (two Wyckoff positions) in the 4(8)-6 structure, as illustrated in Fig. 1(b). Corresponding to these two kinds of $\mathrm{Bi}$ atoms, denoted $\mathrm{Bi}(1)$ and $\mathrm{Bi}(2)$ in the following, there are two buckling heights, $h_{1}=1.579 \AA$ and $h_{2}=2.014 \AA$. The buckling heights of the hexagonal $(1.737-\AA)$ and square $(1.757-\AA$ ) Bi bilayers are in between the two heights of the 4(8)-6 Bi bilayer. The Bi atoms in the 4(8)- 6 bilayer are connected by four different bonds [see Fig. 1(a)], the lengths of which are listed in Table I. The length $d_{1}$ of the bond shared by the six- and eight-atom rings is about $3.046 \AA$, which is practically the same as the bond length in the buckled hexagonal Bi bilayer (3.046 $\AA$ ) [11-14]. The length $d_{2}$ of the bond shared by four- and six-atom rings is about $3.055 \AA$, which is slightly larger than $d_{1}$. The bond length $d_{3}$ shared by the four-atom and eight-atom rings is about $3.079 \AA$, which is larger than the reported bond length (3.059 $\AA$ ) shared by the four-atom and eight-atom rings in the square Bi bilayer [24]. The bond length $d_{4}$ shared by the eight- and eight-atom rings is about $3.043 \AA$, which is nearly the same as the reported bond length $(3.044 \AA)$ shared by the eight- and eight-atom rings in the square Bi bilayer [24]. We can therefore conclude that the proposed structure is formed by an only slightly distorted combination of the square and hexagonal bilayer structures. Similar results are obtained for the 4(8)-6 Sb and As bilayers, although the structure parameters of $\mathrm{Sb}$ and As are smaller than that of the 4(8)-6 Bi bilayer (see Table I). This is in accordance with the general expectation that the lighter the atoms are, the smaller the structure parameters become.

Next, let us focus on the stability of the 4(8)-6 bilayers. To this end, we define the formation energy with respect to the hexagonal bilayer as

$$
\Delta E=\left(E_{\text {total }}-N_{\text {atom }} \times \mu_{\text {atom }}\right) / N_{\text {atom }},
$$

where $E_{\text {total }}$ is the total energy of the 4(8)-6 bilayer, $N_{\text {atom }}$ is the total number of atoms in the crystal structure, and $\mu_{\text {atom }}$ is the energy per atom calculated for the hexagonal honeycomb structure. Starting from $\mathrm{Bi}, \Delta E$ increases with decreasing atomic number. The formation energy for $\mathrm{Bi}$ (52.8 $\mathrm{meV} /$ atom) is the lowest, while $\Delta E$ for $\mathrm{Sb}$ and, especially, As becomes somewhat higher. For comparison, the formation energy of a 4(8)-6 P bilayer was also calculated, and it was found to be even higher ( $76.8 \mathrm{meV} /$ atom). Furthermore, the phonon spectrum of the $\mathrm{P}$ bilayer exhibits imaginary frequency modes, indicating its dynamical instability. Therefore, we do not consider the 4(8)-6 P bilayer in this work. For Bi, the formation energy of the 4(8)-6 Bi bilayer (52.8 meV/atom) is significantly lower than that of a square Bi bilayer (80.6 $\mathrm{meV} /$ atom) [24]. A similar behavior can be observed in 2D C allotropes, where the formation energy decreases with increasing number of $C$ hexagons. In our case, the 4(8)-6 bilayers should be more stable than the reported square bilayers due to the larger number of hexagons [24,25]. To investigate the dynamical stability of the 4(8)-6 bilayers, their phonon spectrum along the high-symmetry lines in the Brillouin zone is calculated from first principles using a supercell approximation [see Fig. 1(c)]. It is shown that the 4(8)-6 bilayers of $\mathrm{Bi}, \mathrm{Sb}$, and $\mathrm{As}$ are all dynamically stable, because no imaginary frequency modes are observed in their phonon spectrum.

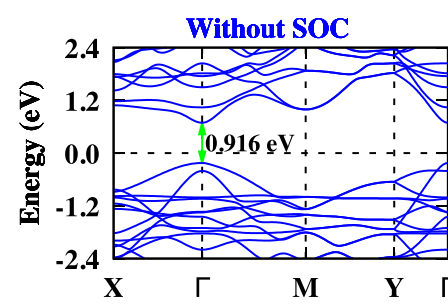

(a) $\mathbf{B i}$

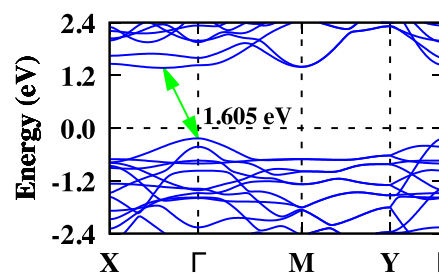

(c) $\mathrm{Sb}$

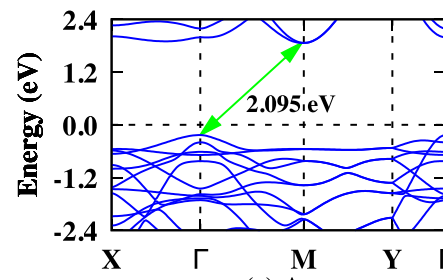

(e) As

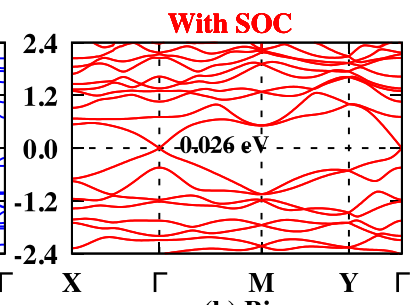

(b) $\mathbf{B i}$

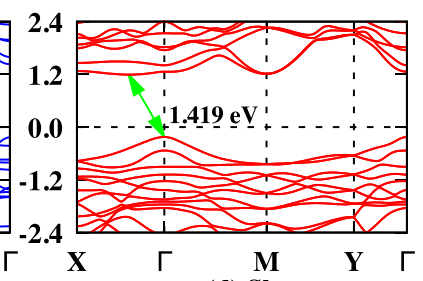

(d) $\mathrm{Sb}$

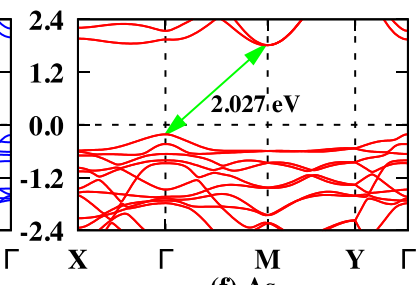

(f) As
FIG. 2. Band structures of (a) 4(8)-6 Bi, (c) 4(8)-6 Sb, and (e) 4(8)-6 As bilayers without SOC; band structures of (b) $4(8)-6 \mathrm{Bi}$, (d) 4(8)-6 SB, and (f) 4(8)-6 As bilayers with SOC. Band gaps are shown. Band structures are calculated at the HSE06 level.

\section{B. Electronic band structures}

As mentioned in Sec. I, 2D materials often have interesting electronic properties. The electronic band structures of the investigated 4(8)-6 bilayers at the HSE06 level are shown in Fig. 2. Figures 2(a), 2(c) and 2(e) (left column) were calculated without SOC, and Figs. 2(b), 2(d) and 2(f) (right column) were calculated with SOC. Let us consider the 4(8)-6 Bi bilayer first. It has a direct band gap of $0.916 \mathrm{eV}$ at the $\Gamma$ point without SOC, which is similar to that of the square $(0.91 \mathrm{eV})$ but larger than that of the hexagonal Bi bilayer $(0.803 \mathrm{eV})[24,54]$. With the inclusion of SOC, the 4(8)-6 Bi bilayer retains its direct band gap, which is similar to the case of the hexagonal bilayer, which also retains a direct band gap with SOC at the HSE06 level [54] The value of the direct band gap becomes $0.026 \mathrm{eV}$, which is smaller than that of the hexagonal/square Bi bilayer [24,54].

On the other hand, the 4(8)-6 Sb/As bilayer has indirect band gaps with and without SOC at the HSE06 level. Differently from the 4(8)-6 Bi bilayers, the SOC effect has little effect on the value of the band gap and the indirect property of the band gap is not changed. Therefore, we focus on the band structures without SOC of the two bilayers. The 4(8)-6 $\mathrm{Sb}$ bilayer has an indirect band gap $(1.605 \mathrm{eV})$, while the 4(8)-6 As bilayer has a larger indirect band gap (2.095 eV). Similarly to the indirect band gap of the hexagonal $\mathrm{Sb} / \mathrm{As}$ bilayer $(2.28 \mathrm{eV} / 2.49 \mathrm{eV})$ [10], the indirect band-gap property is kept and only the value of the band gap is smaller. It has been reported that the hexagonal $\mathrm{Sb} / \mathrm{As}$ bilayer is a promising material for application in optoelectronics [10]. Due to the similarity of the band structure to the hexagonal $\mathrm{Sb} / \mathrm{As}$ bilayer, 
we conclude that the 4(8)-6 Sb/As bilayer can also be applied in nanoscale semiconducting devices. Although 4(8)-6 Bi, Sb, and As bilayers have substantially different band gaps, the regular pattern that the gap values increase with decreasing atomic number is similar to that for hexagonal/square group- $\mathrm{V}$ bilayers $[10,25]$.

For comparison, the band structure at the PBE level is supplied in the Supplemental Material [55]. It is well known that PBE calculations often underestimate the band gap of semiconductors or insulators, which can be confirmed in the band structures without and with SOC of the 4(8)-6 Sb/As bilayer and the band structure without SOC of the 4(8)-6 Bi bilayer. However, the situation is the opposite in the band structure with SOC of the 4(8)-6 Bi bilayer, which is due to the special band structure induced by the SOC effect at the $\Gamma$ point. In the square and hexagonal Bi bilayers, we can also observe the value of the SOC band gap decreasing from the PBE calculations to the HSE06 calculations [24,54].

\section{Topological properties}

Since electronic band gaps with SOC effect of hexagonal and square $\mathrm{Bi}$ bilayers have been shown to be nontrivial $[13,24,25]$, we investigate the topological properties of the 4(8)-6 Bi bilayers here. Note that structures containing $\mathrm{Bi}$ atoms are often reported to be topologically nontrivial due to the strong intrinsic SOC of Bi. To investigate the topological properties of the 4(8)-6 $\mathrm{Bi}$ bilayer, we calculated the $Z_{2}$ topological invariant by Wilson loop methods in the spectrum of the WCCs [55]. In addition to the Wilson loop methods, we also calculated the $Z_{2}$ invariants by parity analysis because the 4(8)-6 Bi bilayer has inversion symmetry. Following Fu et al. [56], the $Z_{2}$ topological invariant $(v)$ in systems with time-reversal symmetry and inversion symmetry can be obtained by

$$
(-1)^{v}=\prod_{i=1}^{4} \delta\left(K_{i}\right), \quad \delta\left(K_{i}\right)=\prod_{m=1}^{N} \xi_{2 m}^{i},
$$

with $K_{i}$ the TRIMs, $\xi= \pm 1$ the parity eigenvalue of the wave function, $\delta\left(K_{i}\right)$ the product of the parity eigenvalues at the TRIM, and $N$ the total number of degenerate occupied bands. In our case $K_{i}$ is $\Gamma, X, Y$, or $M$. The $Z_{2}$ topological invariant of the 4(8)-6 bilayer equals 1 , which proves its nontrivial nature. We list the results of the parity eigenvalues in Table II. It is seen that only the Bi bilayer has a nontrivial topological invariant $(v=1)$, with the only difference in parity eigenvalues between 4(8)-6 $\mathrm{Bi}$ and $\mathrm{Sb}$ or As bilayers at the $\Gamma$ point. The parity eigenvalue of -1 at the $\Gamma$ point of the 4(8)-6 Bi bilayer suggests that there is a band inversion at this TRIM (see below). Similarly to the hexagonal and square bilayers, only the $\mathrm{Bi}$

TABLE II. Parity eigenvalues at the four TRIMs $(\Gamma, X, Y, M)$ and $Z_{2}$ topological invariants $(v)$ of the $4(8)-6 \mathrm{Bi}, \mathrm{Sb}$, and As bilayers.

\begin{tabular}{lccccc}
\hline \hline Element & $\Gamma$ & $X$ & $Y$ & $M$ & $v$ \\
\hline $\mathrm{Bi}$ & -1 & 1 & -1 & -1 & 1 \\
$\mathrm{Sb}$ & 1 & 1 & -1 & -1 & 0 \\
$\mathrm{As}$ & 1 & 1 & -1 & -1 & 0 \\
\hline \hline
\end{tabular}

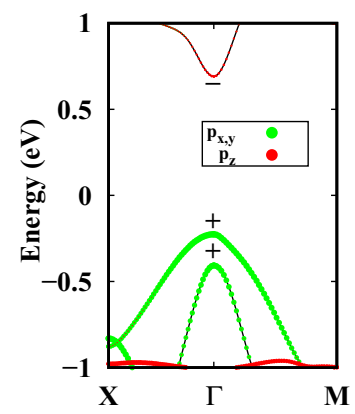

(a) Without SOC

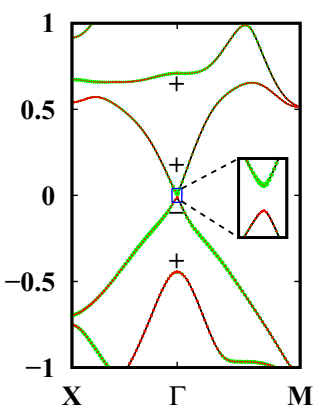

(b) With SOC
FIG. 3. Orbital-projected band structures of the 4(8)-6 Bi bilayer: (a) without SOC and (b) with SOC. The symbol size indicates the contribution weight: a larger dot means a higher contribution; a smaller dot, a lower contribution. \pm indicates an even- or odd-parity eigenvalue. Green, $p_{x, y}\left(p_{x}\right.$ and $\left.p_{y}\right)$ orbitals; red, $p_{z}$ orbitals.

bilayer has a topologically nontrivial band gap, while $\mathrm{Sb}$ and As bilayers have trivial band gaps [13,25].

To find the origin of the topologically nontrivial nature of the 4(8)-6 Bi bilayer, we investigate the band inversion by the orbital-projected band structures as shown in Fig. 3. We can see that SOC plays an important role in the inversion of the states with $p_{x, y^{-}}$and $p_{z}$-orbital character: without SOC at the $\Gamma$ point, the $p_{x, y}$ orbitals contribute the most to the highest occupied band and the $p_{z}$ orbitals contribute the most to the lowest unoccupied band, but the situation is reversed when SOC is included. This is also verified by the reversal of parity eigenvalues between the highest occupied band and the lowest unoccupied band at the $\Gamma$ point. Note that the observed band inversion found here is similar to the case of hexagonal and square Bi bilayers, whose band inversion also occurs between the $p_{x, y}$ and the $p_{z}$ orbitals $[13,24,25]$. The band inversion indicates that there must be a gap closing with corresponding formation of a Dirac cone when the SOC is continuously turned on $[24,57]$. To find the Dirac cone, continuous variations of SOC strengths $\lambda$ were applied to calculate the corresponding

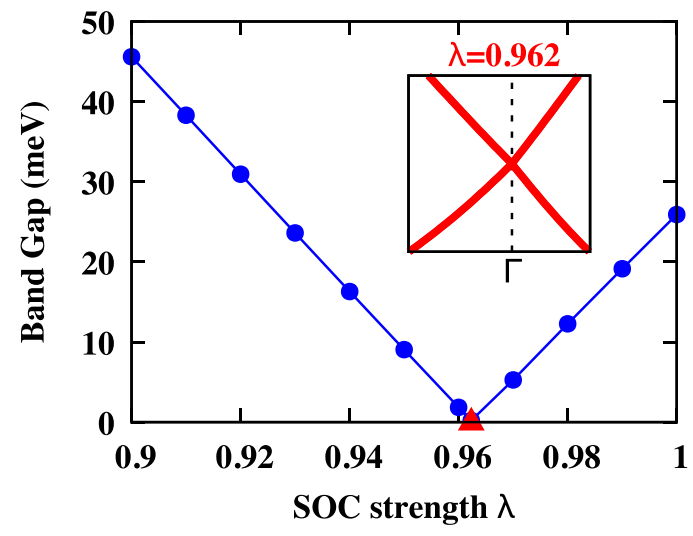

FIG. 4. Variation of the band gap in 4(8)-6 Bi bilayer as a function of the SOC strength $\lambda$. The red triangle indicates the critical point at $\lambda=0.962$. Inset: Gapless Dirac point at the $\Gamma$ point for $\lambda=0.962$. The band crossing with a Dirac point indicates that there is a topological phase transition with continuous tuning of the SOC strength $\lambda$. 


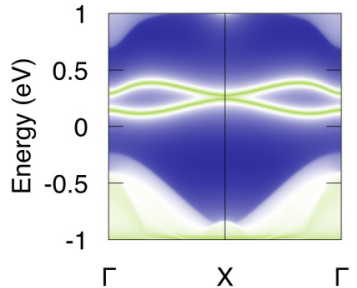

(a) Without SOC

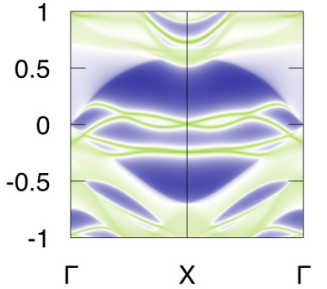

(b) With SOC
FIG. 5. Edge states of a 4(8)-6 Bi bilayer: (a) without SOC and (b) with SOC.

band gaps of the 4(8)-6 Bi bilayer. As shown in Fig. 4, for $\lambda<0.962$, the band gaps decrease as $\lambda$ increases; for $\lambda>0.962$, the band gaps begin to increase as $\lambda$ increases; and for $\lambda=0.962$, there is a zero-gap state. Therefore, the band structure for $\lambda=0.962$ is plotted in the inset, and a gap closing with a Dirac point at the $\Gamma$ point can be observed.

Besides the nonzero $Z_{2}$ topological invariant and the observed band inversion, the existence of gapless edge states is another prominent feature of QSH insulators. According to the bulk-edge correspondence in topological insulators, a nontrivial topological invariant $(v=1)$ indicates the presence of topologically protected edge states at the edges of the material. The calculated edge states of the 4(8)-6 Bi bilayer are shown in Fig. 5. In Fig. 5(a), it is shown that there are edge states in the band gap without SOC, but these edge states do not bridge the band gap, which indicates that they are trivial. However, including SOC, two oppositely propagating gapless edge states appear in the bulk gap that connect the conduction and valence bands and cross at the TRIM ( $X$ point) as shown in Fig. 5(b).

\section{Structural tunability}

As discussed above, the formation energy decreases with an increasing number of six-atom rings in the system. In this section we investigate how the properties of the $4(8)-6 \mathrm{Bi}$ bilayer can be tuned by changing the number of hexagons. This is done in a systematic way by increasing the number of six-connected lines which connect the centers of six-atom rings in the $x$ direction. Taking $\mathrm{Bi}$ as an example, we show three such structures in Fig. 6. As indicated by the dashed blue lines, the number of six-connected lines is two, three, and four in these structures, and they are correspondingly
TABLE III. Optimized structure parameters of 4(8)-6-6, 4(8)-66-6, and 4(8)-6-6-6-6 Bi bilayers. $a(b)$ is the lattice constant in the $x(y)$ direction; $h$ is the averaged buckling height.

\begin{tabular}{lccc}
\hline \hline Structure & $\begin{array}{c}a \\
(\AA)\end{array}$ & $\begin{array}{c}b \\
(\AA)\end{array}$ & $\begin{array}{c}h \\
(\AA)\end{array}$ \\
\hline $4(8)-6-6$ & 7.778 & 17.493 & 1.735 \\
$4(8)-6-6-6$ & 7.708 & 21.834 & 1.734 \\
$4(8)-6-6-6-6$ & 7.661 & 26.188 & 1.734 \\
\hline \hline
\end{tabular}

named 4(8)-6-6, 4(8)-6-6-6, and 4(8)-6-6-6-6. The calculated structure parameters of the three structures are listed in Table III. Due to the similar structure along the $x$ direction compared to 4(8)-6, the lattice constants of the expanded structures in the $x$ direction are similar $(7.919,7.778,7.708$, and $7.661 \AA$ ) and are in between those of the square and those of the hexagonal structures. We can easily understand this: the larger the number of six-atom rings becomes, the closer to the hexagonal $\mathrm{Bi}$ bilayer the expanded structure gets. There is now a larger variety of different $\mathrm{Bi}$ atoms, corresponding to various buckling heights, so we compare the averaged buckling heights. These are 1.724, 1.735, 1.734, and $1.734 \AA$, respectively. Note that these averaged buckling heights are larger than the buckling height of $1.71 \AA$ found in the hexagonal Bi bilayer [14,58] and smaller than the $1.76 \AA$ in the square Bi bilayer [24,25].

We also calculated the energy per atom in all these structures and compared them to the hexagonal and square Bi bilayers which have been studied before [14,24,25,58]. As shown in Fig. 7, the hexagonal Bi bilayer has the lowest energy, which implies that it is the most stable of all these structures. On the other hand, the square Bi bilayer has the highest energy of all. The energy per atom of the new proposed structures varies monotonously between these two limiting structures and converges to the energy of the hexagonal bilayer as more hexagons are included.

The electronic band structures of the 4(8)-6-6, 4(8)-6-6-6, and 4(8)-6-6-6-6 Bi bilayers at the HSE06 level are shown in Fig. 8. As shown in Figs. 8(a), 8(c) and 8(e) (left column), the direct band gaps of the three structures calculated without SOC are $0.820,0.880$, and $0.890 \mathrm{eV}$, which are smaller than that of the 4(8)-6 Bi bilayer $(0.916 \mathrm{eV})$. After including the SOC, as shown in Figs. 8(b), 8(d) and 8(f) (right column), the direct band gaps of the three structures are $0.303,0.237$,

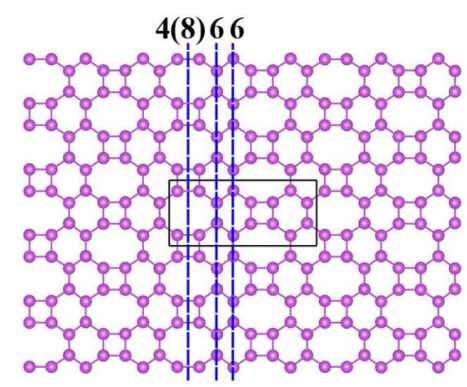

(a) 4(8)-6-6

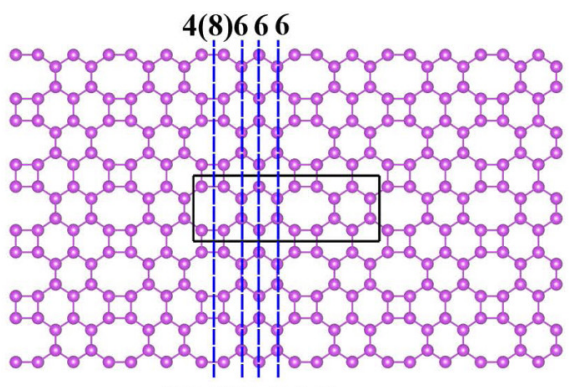

(b) 4(8)-6-6-6

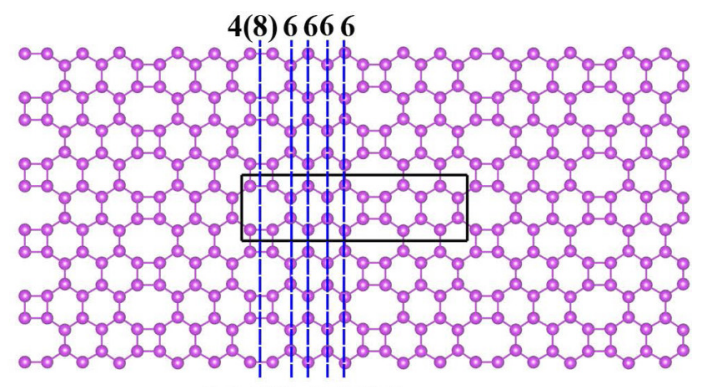

(c) $4(8)-6-6-6-6$

FIG. 6. Tunable structures: (a) 4(8)-6-6, (b) 4(8)-6-6-6, and (c) 4(8)-6-6-6-6 Bi bilayers. Dashed blue lines represent the 4(8)-centerconnected and 6-center-connected lines. 


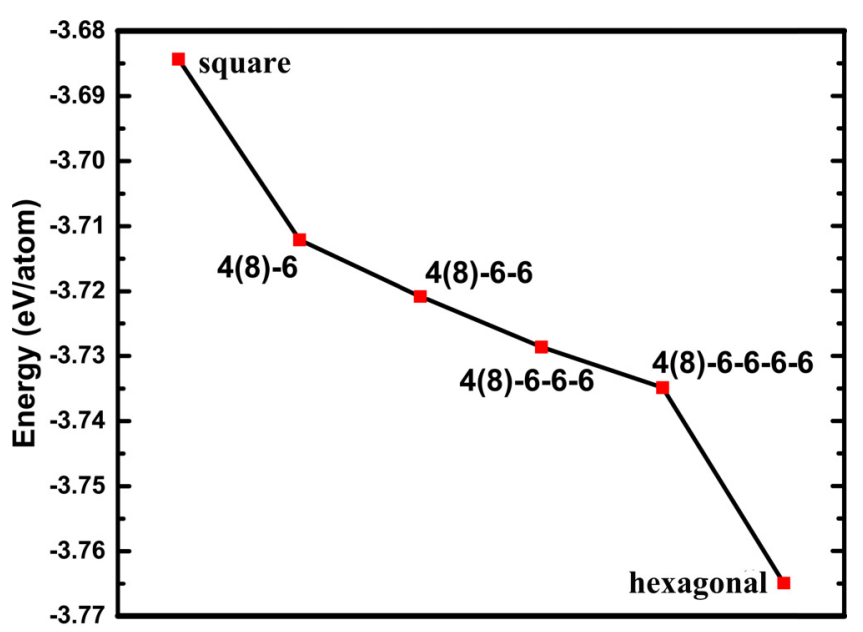

Structures

FIG. 7. Energy per atom of 4(8)-6, 4(8)-6-6, 4(8)-6-6-6, and 4(8)-6-6-6-6 Bi bilayers.

and $0.311 \mathrm{eV}$, which are much larger than that of the 4(8)-6 Bi bilayer $(0.026 \mathrm{eV})$. For the three structures, their band structures at the PBE level are reported in the Supplemental Material [55]. Similarly to the band structure of the 4(8)-6 Bi bilayer with SOC, they also show the value of the band gap decreasing from the PBE calculations to the HSE calculations. Since the SOC band gap of the 4(8)-6 Bi bilayer is nontrivial, we calculated the $Z_{2}$ topological invariant of the new three $\mathrm{Bi}$

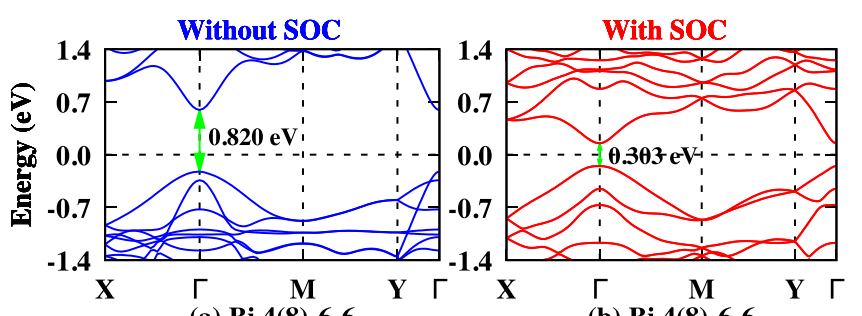
(a) $\mathrm{Bi}$ 4(8)-6-6

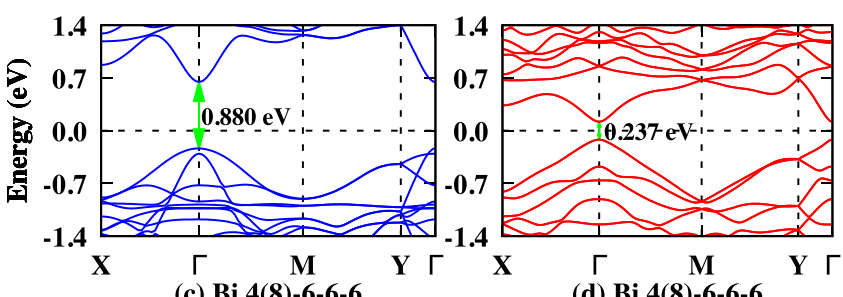
(c) $\mathrm{Bi}$ 4(8)-6-6-6

(d) $\mathrm{Bi}$ 4(8)-6-6-6

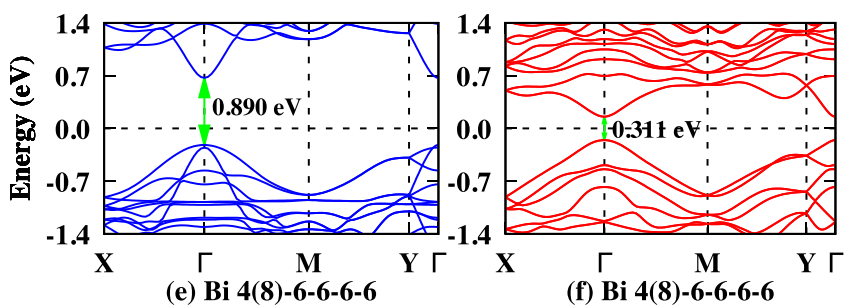

FIG. 8. Band structures of (a) 4(8)-6-6, (c) 4(8)-6-6-6, and (e) 4(8)-6-6-6-6 Bi bilayers without SOC; band structures of (b) 4(8)-6-6, (d) 4(8)-6-6-6, and (f) 4(8)-6-6-6-6 Bi bilayers with SOC. Band gaps are shown. Band structures are calculated at the HSE06 level.

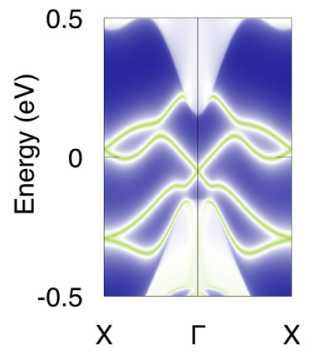

(a) $4(8)-6-6$

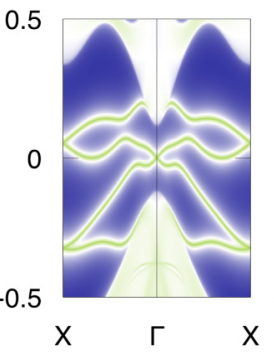

(b) $4(8)-6-6-6$

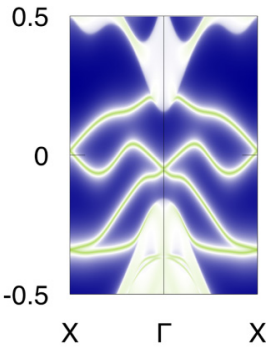

(c) $4(8)-6-6-6-6$
FIG. 9. Nontrival edge states of the structures: (a) 4(8)-6-6, (b) 4(8)-6-6-6, and (c) 4(8)-6-6-6-6 Bi bilayers.

bilayers, and all the $Z_{2}$ values are equal to 1 , which confirms that they are all 2D topological insulators [55]. It can be concluded that the value of the nontrivial SOC band gap will approach that of the hexagonal Bi bilayer upon the inclusion of more Bi hexagons $[13,54]$. To further confirm their topological properties, the nontrivial edge states of 4(8)-6-6, 4(8)-6-6-6, and 4(8)-6-6-6-6 Bi bilayers are shown in Fig. 9, and edge Dirac cones are clearly visible. The topological edge states of the three structures appear to be very similar.

In general, we can conclude that as the number of six-atom rings increases, the energy, electronic structure, and nontrivial band gap approach those of the hexagonal Bi bilayer. At the same time, the lines of four- and eight-atom rings can be regarded as line defects in the hexagonal $\mathrm{Bi}$ bilayer. By introducing such line defects, it is possible to tune the properties of the hexagonal Bi bilayer.

\section{CONCLUSIONS}

Using first-principles calculations, we propose a new stable 4(8)-6 model for the group-V elements ( $\mathrm{Bi}, \mathrm{Sb}, \mathrm{As}$ ), which enrich the family of $2 \mathrm{D}$ materials. Their formation energy compares favorably to that of the square bilayer, while our phonon calculations confirm their dynamical stability. The trivial ( $\mathrm{Sb}, \mathrm{As}$ ) and nontrivial (Bi) band gaps make the group-V 4(8)-6 structures promising candidates for application in future nanodevices. The nontrivial topological phase of the $\mathrm{Bi} 4(8)-6$ structure is demonstrated by the calculations of the $Z_{2}$ topological invariant, band inversion, and edge states. Moreover, the 4(8)-6 model allows for property tuning by changing the ratio of hexagons in the structure. In the case of $\mathrm{Bi}$, we investigated three such models, namely, 4(8)-6-6, 4(8)6-6-6, and 4(8)-6-6-6-6, and found that they are all topological insulators. As the number of hexagons increases, the energy, electronic structure, and nontrivial band gap approach those of the hexagonal $\mathrm{Bi}$ bilayer. In the dilute limit, the lines of four-atom and eight-atom rings can be regarded as line defects in the hexagonal Bi bilayer that can be used to tune the properties of the hexagonal Bi bilayer.

\section{ACKNOWLEDGMENTS}

This work is supported by Ministry of Science and Technology of China (MOST) (Grant No. 2016YFA0301604), National Natural Science Foundation of China (NSFC) (No. 11574008), the Thousand-Young-Talent Program of China, and the Fonds voor Wetenschappelijk Onderzoek (FWO- 
V1). The computational resources and services used in this work were provided by the VSC (Flemish Supercomputer Center), funded by the Research Foundation - Flanders (FWO) and the Flemish Government - department EWI, and the National Supercomputing Center in Tianjin, funded by the Collaborative Innovation Center of Quantum Matter.
[1] K. S. Novoselov, A. K. Geim, S. Morozov, D. Jiang, Y. Zhang, S. V. Dubonos, I. V. Grigorieva, and A. A. Firsov, Science 306, 666 (2004).

[2] S. J. Kim, K. Choi, B. Lee, Y. Kim, and B. H. Hong, Annu. Rev. Mater. Res. 45, 63 (2015).

[3] B. Feng, Z. Ding, S. Meng, Y. Yao, X. He, P. Cheng, L. Chen, and K. Wu, Nano Lett. 12, 3507 (2012).

[4] P. Vogt, P. D. Padova, C. Quaresima, J. Avila, E. Frantzeskakis, M. C. Asensio, A. Resta, B. Ealet, and G. L. Lay, Phys. Rev. Lett. 108, 155501 (2012).

[5] D. Chiappe, C. Grazianetti, G. Tallarida, M. Fanciulli, and A. Molle, Adv. Mater. 24, 5088 (2012); S. Cahangirov, M. Topsakal, E. Aktürk, H. Şahin, and S. Ciraci, Phys. Rev. Lett. 102, 236804 (2009).

[6] D. Chiappe, E. Scalise, E. Cinquanta, C. Grazianetti, B. van den Broek, M. Fanciulli, M. Houssa, and A. Molle, Adv. Mater. 26, 2096 (2014).

[7] E. Bianco, S. Butler, S. Jiang, O. D. Restrepo, W. Windl, and J. E. Goldberger, ACS Nano 7, 4414 (2013).

[8] M. Derivaz, D. Dentel, R. Stephan, M.-C. Hanf, A. Mehdaoui, P. Sonnet, and C. Pirri, Nano Lett. 15, 2510 (2015).

[9] F.-f. Zhu, W.-j. Chen, Y. Xu, C.-1. Gao, D.-d. Guan, C.-h. Liu, D. Qian, S.-C. Zhang, and J.-f. Jia, Nat. Mater. 14, 1020 (2015).

[10] S. Zhang, Z. Yan, Y. Li, Z. Chen, and H. Zeng, Angew. Chem. Int. Ed. 54, 3112 (2015).

[11] A. Takayama, T. Sato, S. Souma, T. Oguchi, and T. Takahashi, Phys. Rev. Lett. 114, 066402 (2015).

[12] I. K. Drozdov, A. Alexandradinata, S. Jeon, S. Nadj-Perge, H. Ji, R. J. Cava, B. A. Bernevig, and A. Yazdani, Nat. Phys. 10, 664 (2014).

[13] X. Li, H. Liu, H. Jiang, F. Wang, and J. Feng, Phys. Rev. B 90, 165412 (2014).

[14] Z. Liu, C.-X. Liu, Y.-S. Wu, W.-H. Duan, F. Liu, and J. Wu, Phys. Rev. Lett. 107, 136805 (2011).

[15] F. Yang, L. Miao, Z. F. Wang, M.-Y. Yao, F. Zhu, Y. R. Song, M.-X. Wang, J.-P. Xu, A. V. Fedorov, Z. Sun, G. B. Zhang, C. Liu, F. Liu, D. Qian, C. L. Gao, and J.-F. Jia, Phys. Rev. Lett. 109, 016801 (2012)

[16] T. Hirahara, G. Bihlmayer, Y. Sakamoto, M. Yamada, H. Miyazaki, S.-i. Kimura, S. Blügel, and S. Hasegawa, Phys. Rev. Lett. 107, 166801 (2011).

[17] T. Hirahara, N. Fukui, T. Shirasawa, M. Yamada, M. Aitani, H. Miyazaki, M. Matsunami, S. Kimura, T. Takahashi, S. Hasegawa, and K. Kobayashi, Phys. Rev. Lett. 109, 227401 (2012).

[18] J. L. Zhang, S. Zhao, C. Han, Z. Wang, S. Zhong, S. Sun, R. Guo, X. Zhou, C. D. Gu, K. D. Yuan, Z. Li, and W. Chen, Nano Lett. 16, 4903 (2016).

[19] S. H. Zhang, J. Zhou, Q. Wang, X. S. Chen, Y. Kawazoe, and P. Jena, Proc. Natl. Acad. Sci. USA 112, 2372 (2015).

[20] Y. Liu, G. Wang, Q. Huang, L. Guo, and X. Chen, Phys. Rev. Lett. 108, 225505 (2012).
[21] Z. Wang, X.-F. Zhou, X. Zhang, Q. Zhu, H. Dong, M. Zhao, and A. R. Oganov, Nano Lett. 15, 6182 (2015).

[22] B. G. Kim and H. J. Choi, Phys. Rev. B 86, 115435 (2012).

[23] A. Wang, L. Li, X. Wang, H. Bu, and M. Zhao, Diamond Relat. Mater. 41, 65 (2014).

[24] L. Kou, X. Tan, Y. Ma, H. Tahini, L. Zhou, Z. Sun, D. Aijun, C. Chen, and S. C. Smith, 2D Mater. 2, 45010 (2015).

[25] P. Li and W. Luo, Sci. Rep. 6, 25423 (2016).

[26] X.-L. Qi and S.-C. Zhang, Rev. Mod. Phys. 83, 1057 (2011).

[27] M. Z. Hasan and C. L. Kane, Rev. Mod. Phys. 82, 3045 (2010).

[28] B. A. Bernevig and S.-C. Zhang, Phys. Rev. Lett. 96, 106802 (2006).

[29] C. L. Kane and E. J. Mele, Phys. Rev. Lett. 95, 226801 (2005).

[30] Y. Yao, F. Ye, X.-L. Qi, S.-C. Zhang, and Z. Fang, Phys. Rev. B 75, 041401 (2007).

[31] B. A. Bernevig, T. L. Hughes, and S.-C. Zhang, Science 314, 1757 (2006).

[32] I. Knez, R.-R. Du, and G. Sullivan, Phys. Rev. Lett. 107, 136603 (2011).

[33] M. König, S. Wiedmann, C. Brüne, A. Roth, H. Buhmann, L. W. Molenkamp, X.-L. Qi, and S.-C. Zhang, Science 318, 766 (2007).

[34] L. Li, X. Zhang, X. Chen, and M. Zhao, Nano Lett. 15, 1296 (2015).

[35] X. Chen, L. Li, and M. Zhao, Phys. Chem. Chem. Phys. 17, 16624 (2015).

[36] X. Chen, L. Li, and M. Zhao, RSC Adv. 5, 72462 (2015).

[37] M. Zhao, X. Zhang, and L. Li, Sci. Rep. 5, 16108 (2015).

[38] M. Zhao, X. Chen, L. Li, and X. Zhang, Sci. Rep. 5, 8441 (2015).

[39] Z. Song, C.-C. Liu, J. Yang, J. Han, M. Ye, B. Fu, Y. Yang, Q. Niu, J. Lu, and Y. Yao, NPG Asia Mater. 6, e147 (2014).

[40] C.-C. Liu, S. Guan, Z. Song, S. A. Yang, J. Yang, and Y. Yao, Phys. Rev. B 90, 085431 (2014).

[41] G. Kresse and J. Furthmüller, Phys. Rev. B 54, 11169 (1996).

[42] G. Kresse and J. Hafner, Phys. Rev. B 48, 13115 (1993).

[43] G. Kresse and D. Joubert, Phys. Rev. B 59, 1758 (1999).

[44] J. P. Perdew, K. Burke, and M. Ernzerhof, Phys. Rev. Lett. 77, 3865 (1996).

[45] J. Heyd, G. E. Scuseria, and M. Ernzerhof, J. Chem. Phys. 118 , 8207 (2003).

[46] J. Heyd, G. E. Scuseria, and M. Ernzerhof, J. Chem. Phys. 124, 219906 (2006)

[47] A. Togo and I. Tanaka, Scr. Mater. 108, 1 (2015).

[48] A. A. Soluyanov and D. Vanderbilt, Phys. Rev. B 83, 235401 (2011).

[49] R. Yu, X. L. Qi, A. Bernevig, Z. Fang, and X. Dai, Phys. Rev. B 84, 075119 (2011).

[50] D. Gresch, G. Autès, O. V. Yazyev, M. Troyer, D. Vanderbilt, B. A. Bernevig, and A. A. Soluyanov, Phys. Rev. B 95, 075146 (2017). 
[51] A. A. Mostofi, J. R. Yates, G. Pizzi, Y.-S. Lee, I. Souza, D. Vanderbilt, and N. Marzari, Comput. Phys. Commun. 185, 2309 (2014).

[52] M. P. L. Sancho, J. M. L. Sancho, J. M. L. Sancho, and J. Rubio, J. Phys. F: Met. Phys. 15, 851 (1985).

[53] Q. Wu, S. Zhang, H.-F. Song, M. Troyer, and A. A. Soluyanov, arXiv:1703.07789.

[54] E. Aktürk, O. U. Aktürk, and S. Ciraci, Phys. Rev. B 94, 014115 (2016).
[55] See Supplemental Material at http://link.aps.org/supplemental/ 10.1103/PhysRevB.96.035123 for the electronic band structures at the PBE level and the evolution of WCCs for calculating the $Z_{2}$ topological invariant at the HSE06 level.

[56] L. Fu and C. L. Kane, Phys. Rev. B 76, 045302 (2007).

[57] L. Li, O. Leenaerts, X. Kong, X. Chen, M. Zhao, and F. M. Peeters, Nano Res. 10, 2168 (2017).

[58] L. Chen, Z. F. Wang, and F. Liu, Phys. Rev. B 87, 235420 (2013). 